\title{
Die Rolle elternbeurteilter exekutiver Funktionen zur Schulleistungsprognose
}

\author{
Eva Michel (ㅇ, Lena Söll, Sabine Molitor \\ Lehrstuhl für Psychologie IV, Universität Würzburg
}

\begin{abstract}
Zusammenfassung: Einleitung: Unter dem Begriff exekutive Funktionen (EF) werden häufig die Komponenten Inhibition, kognitive Flexibilität und Aktualisierung von Arbeitsgedächtnisrepräsentationen subsumiert. EF sind bereichsübergreifende Prädiktoren schulischer Leistungen. Verschiedene Operationalisierungen derselben Komponente, z. B. Performanztests und Elterneinschätzungen, zeigen häufig nur geringe Interkorrelationen. Die Methoden scheinen unterschiedliche Aspekte einer Komponente zu erfassen, daher könnte eine Kombination zur Vorhersage schulischer Leistungen sinnvoll sein. Methode: $N$ = 96 Erst- und Zweitklässler_innen mit und ohne Entwicklungsauffälligkeiten wurden mittels EF-Performanztests und Schulleistungstests zu Mathematik und Lesen untersucht. Per Fragebogen wurden elternbeurteilte EF und als Kontrollvariablen der sozioökonomische Status (SÖS) und das Vorliegen von Merkmalen einer Aufmerksamkeitsdefizit-Hyperaktivitätsstörung (ADHS) erfasst. Ergebnisse: Elternbeurteilungen hatten über die Performanztests hinaus einen bedeutsamen Vorhersagewert für die Mathematik- und Leseleistung. Der Einfluss von Alter, SÖS und ADHS-Merkmalen wurde kontrolliert. Diskussion: Die kombinierte Anwendung beider Erfassungsmethoden scheint somit vorteilhaft für die Prognose schulischer Leistungen und die Prävention von Schulleistungsproblemen.
\end{abstract}

Schlüsselwörter: Exekutive Funktionen, Prädiktoren von Schulleistungen, ADHS, Mathematik, Lesen

The Role of Parent-Rated Executive Functions as Predictors of School Performance

Abstract: Introduction: The term „executive functions“ (EF) often embraces inhibition, cognitive flexibility and working memory updating. EF are domain-general predictors of scholastic performance. Different operationalisations of the same component, e.g., individual tests and parent ratings, often show weak interrelations. This indicates that different methods measure different aspects of an EF component. A combination of operationalisation methods might thus enhance the prediction of school performance. Method: $N=961^{\text {st }}$ and $2^{\text {nd }}$ graders with typical/atypical development were tested with individual EF tests, as well as mathematics and reading tests. Parent-rated EF, socio-economic status (SES) and ADHD symptoms (as control variables) were assessed with a questionnaire. Results: Parent ratings improve the prediction of mathematics and reading performance over and above individual tests. Age, SES and ADHD symptoms were controlled. Discussion: Combining both EF operationalisations provides benefits for prognosis and prevention of scholastic achievement problems.

Keywords: Executive functions, predictors of scholastic performance, ADHD, mathematics, reading

\section{Einleitung}

Aufgrund der großen Bedeutung des Schulerfolgs für das emotionale Wohlbefinden von Kindern und Jugendlichen und den späteren Berufserfolg sind Faktoren, die schulische Leistungen beeinflussen, bereits lange im Forschungsfokus. Die Prognose schulischer Leistungen ist eins der zentralen Themen der Pädagogischen Psychologie. Obwohl sich interindividuelle Unterschiede in den schulischen Leistungen bereits relativ früh stabilisieren, und somit die Schulleistung zu einem früheren Zeitpunkt einen hohen Vorhersagewert für die weitere Leistungsentwicklung aufweist, spielen bereichsunspezifische Prädik- toren zusätzlich eine wichtige Rolle, besonders zu Beginn der Beschulung. Für die Früherkennung und Prävention von Schulleistungsproblemen wurde in den letzten Jahren insbesondere die Bedeutung exekutiver Funktionen (EF) hervorgehoben (Bailey, 2007; Best, Miller \& Naglieri, 2011; Visu-Petra, Cheie, Benga \& Miclea, 2011). Diese umfassen kognitive, größtenteils präfrontale Prozesse, die Denken und Verhalten zielgerichtet lenken (Kubesch, Emrich \& Beck, 2011; Otero, Barker \& Naglieri, 2014). Eine möglichst genaue Erfassung dieses heterogenen Konstrukts ist die Voraussetzung, um Aussagen über die Bedeutsamkeit für schulische Leistungen treffen zu können. Meist werden dazu computergestützte Performanztests, 
also individuelle Leistungstests im Labor, eingesetzt. Ein Kritikpunkt hierbei ist die mangelnde ökologische Validität und Verallgemeinerbarkeit auf Alltagssituationen (Barkley \& Fischer, 2011; Isquith, Roth \& Gioia, 2013; Miranda, Colomer, Mercader, Fernandez \& Presentación, 2015). Alltagsnähere Maße sind Beurteilungen durch Eltern und / oder Lehrkräfte (Gioia, Isquith, Retzlaff \& Espy, 2002). Bislang existieren kaum Studien, in denen beide Erfassungsmethoden zur Vorhersage schulischer Leistungen eingesetzt wurden (Dekker, Ziermanns, Spuijt \& Swaab, 2017). In der hier beschriebenen Studie soll daher untersucht werden, inwieweit Performanztests und Elternbeurteilungen der EF für die Vorhersage der Lese- und Mathematikleistung von Erst- und Zweitklässler_innen relevant sind. Als Kontrollvariablen werden -neben dem Alter- der sozioökonomische Status (SÖS) und ADHSSymptome einbezogen. Dadurch kann die relative Bedeutsamkeit der EF unter Kontrolle dieser möglicherweise konfundierten Variablen untersucht werden.

\section{Wissenschaftlicher Hintergrund - Exekutive Funktionen}

EF sind in neuartigen und herausfordernden Situationen relevant, wenn automatisierte Handlungsroutinen nicht ausreichen (Banich, 2009; Drechsler, 2007). Zumeist werden drei Komponenten unterschieden (Diamond, 2013; Miyake \& Friedman, 2012): a) Inhibitionskontrolle, d.h. die Fähigkeit, prädominante Reaktionen bewusst zu unterdrücken (zugunsten situationsangemessenerer Reaktionen); b) Überwachung der Arbeitsgedächtnisinhalte („Updating“) und c) kognitive Flexibilität, d.h. der flexible Wechsel zwischen Lösungswegen und Perspektiven, je nach Aufgabenanforderungen (,Shifting“). Die drei Komponenten sind moderat interkorreliert (Miyake et al., 2000) und bilden die Basis für komplexe Selbstregulationsprozesse (Drechsler, 2007). Bei Grundschulkindern bilden Inhibition und Shifting häufig noch einen kombinierten Faktor (Lee et al., 2012; Van der Ven et al., Kroesbergen, Boom \& Leseman, 2013); allerdings finden sich auch Belege für eine frühe Drei-Faktoren-Struktur (Lehto, Juujärvi. Kooistra \& Pulkkinen, 2003). In vorliegender Studie wurden die drei „klassischen“ Komponenten nach Miyake et al. (2000) erfasst, da die Subsumierung von Prozessen der Inhibition und Interferenzkontrolle, des Arbeitsgedächtnisses und der kognitiven Flexibilität unter den Oberbegriff der EF auch bei Studien mit Grundschulkindern weitgehend Konsens ist. EF sind zentrale Prädiktoren schulischer Leistungen und können diese teils besser vorhersagen als Intelligenzmaße (Michel, Molitor \& Schneider, 2019; Roebers et al.,, 2014; Szücs, Devine, Soltesz, Nobes \& Gabriel., 2014).

\section{Die Rolle der EF für den Leseerwerb}

Neben bereichsübergreifenden Funktionen (z.B. Ausblendung von Störreizen, Umsetzung mehrschrittiger Handlungsanweisungen; Gathercole \& Alloway, 2016) lenken EF Aufmerksamkeitsressourcen beim Leseprozess selbst (z. B. Reynolds, 2000). Im Zwei-Wege-Modell der visuellen Worterkennung (Coltheart, Rastle, Perry, Langdon \& Ziegler, 2001) spielen Arbeitsgedächtnisprozesse vor allem zu Beginn des Schriftspracherwerbs eine wichtige Rolle. Der Sichtwortschatz ist bei Leseanfänger_innen noch klein, sodass Wörter häufig indirekt per BuchstabeLaut-Zuordnung entschlüsselt werden müssen. Erkannte Laute müssen im Arbeitsgedächtnis aufrechterhalten werden, während weitere Buchstaben verarbeitet, in Laute transferiert und verbunden werden (Marx, 2007). Inhibition wird bei der Hemmung optisch oder semantisch ähnlicher Wörter benötigt (Massol, Molinato \& Carreiras, 2015). Kognitive Flexibilität sorgt für die Integration mit Vorwissen (Gerst, Cirino, Fletcher \& Yoshida, 2017). Entsprechend wurden auch metaanalytisch $\mathrm{Zu}$ sammenhänge zwischen EF und Lesefähigkeiten gefunden (Butterfuss \& Kendeou, 2018; Follmer, 2018; Ober, Brooks, Homer \& Rindskopf, 2020).

\section{Die Rolle der EF für den Erwerb mathematischer Kompetenzen}

Bei Rechenoperationen kommen Arbeitsgedächtnisprozesse zur Speicherung und zum Abruf von Teilergebnissen zum Einsatz (Van der Ven, Kroesbergen, Boom \& Leseman, 2012). Inhibitionsprozesse sorgen dafür, dass falsche Rechenmethoden oder -ergebnisse unterdrückt werden (Clements, Sarama \& Germeroth, 2016). Shifting-Prozesse können beim flexiblen Wechsel zwischen verschiedenen Lösungsstrategien oder Rechenoperationen relevant sein (Bull \& Lee, 2014). Die Beteiligung der EF bei mathematischen Leistungen wurde empirisch häufig nachgewiesen (Bull, Espy \& Wiebe, 2008; Bull \& Lee, 2014; Van der Ven et al., 2012; Visu-Petra et al., 2011; Yeniad, Malda, Mesman, van Ijzendoorn \& Pieper, 2013).

\section{Erhebung exekutiver Funktionen: Performanztests und Elternbeurteilungen im Vergleich}

Zwischen Performanztests und Elternbeurteilungen werden häufig nur niedrige Zusammenhänge gefunden (McAuley et al., 2010; Schöfl, Kloo \& Kaufmann, 2015; Silver, 2014; Toplak, West \& Stanovich, 2013). Drei mög- 
liche Erklärungen werden dafür diskutiert: Erstens könnten EF hierarchisch aufeinander aufbauend sein, und die Messmethoden könnten unterschiedliche Stufen erfassen (Barkley \& Fischer, 2011). Untere Stufen sollten selbstgesteuertes Verhalten in kurzen Zeitabschnitten umfassen (z.B. unmittelbar bei der Bearbeitung einer Aufgabe), wie es über Performanztests erfasst wird. Höhere Stufen sollten Fähigkeiten umfassen, die längerfristig im sozialen Alltag notwendig sind; diese werden eher in verhaltensbezogenen Fragebögen erfasst.

Eine zweite Erklärung ist, dass Performanztests die maximale Leistungsfähigkeit erfassen, da das Kind sich z.B. besonders anstrengt, Elternbeurteilungen dagegen eher das Verhalten im Alltag (Toplak et al. 2013).

Und drittens erfassen Performanztests in der Regel EF ohne emotionale Beteiligung, Elternbeurteilungen dagegen EF, mit denen Emotionen reguliert und persönlich relevante Entscheidungen getroffen werden müssen (z.B. Ritter, Perrig, Steinlin \& Everts, 2014).

Aus allen drei Perspektiven sollten beide Erfassungsmethoden zur Schulleistungsprognose relevant sein, da die verschiedenen genannten Aspekte jeweils separat zu Unterschieden in Schulleistungen beitragen sollten.

\section{Vorhersage schulischer Leistungen durch Performanztests und Elternbeurteilungen der EF}

Nur wenige Studien beinhalten beide Erhebungsmethoden (Dekker et al., 2017). Bezüglich der Leseleistung waren sowohl die Arbeitsgedächtnis- und Inhibitionsleistung in Performanztests als auch das Lehrkraft- und Elternrating der EF (BRIEF; Behavior Rating Inventory of Executive Function; Gioia et al., 1998, 2000) bei 5- und 6-jährigen Kindern bedeutsame Prädiktoren (Miranda et al., 2015). Die Performanztests klärten am meisten Varianz auf. Bei älteren Kindern (10-14 Jahre) waren sowohl die BRIEF-Elternversion als auch die Inhibitions- und Arbeitsgedächtnisleistung in Performanztests zur Unterscheidung von unauffälligen Schüler_innen und solchen mit Worterkennungsschwierigkeiten geeignet (Locascio, Mahone, Eason \& Cutting, 2010). Für die Lese- und Mathematikleistung im Alter von 5 bis 18 Jahren waren sowohl Elterneinschätzung als auch Performanztests zu Inhibition und kognitiver Flexibilität prädiktiv (Ten Eycke \& Dewey, 2016).

Bzgl. der Mathematikleistung klärten bei 5- bis 6-jährigen Kindern sowohl (Lehrkraft- und) Elternratings (BRIEF) als auch Performanztests zusätzlich zum IQ einen signifikanten Varianzanteil auf (Zorrilla-Silvestre, Presentación-Herrero \& Gil-Gómez, 2016). Das Lehrkraftrating hatte hier den größten Vorhersagewert, ge- folgt von Elternbeurteilungen und Performanztests. In einer der wenigen Studien, in denen der inkrementelle prädiktive Wert der Elternbeurteilungen über Performanztests hinaus untersucht wurde, erfassten Dekker et al. (2017) die Arbeitsgedächtnis-, kognitive Flexibilitäts- und Inhibitionsleistung von 6- bis 8-jährigen Schüler_innen anhand von Performanztests und der BRIEF-Eltern- und Lehrkraftversion. In drei hierarchischen Regressionsmodellen wurden separat für die drei EF in einem ersten Schritt die Performanztests und in einem zweiten Schritt die Fremdbeurteilungen zur Vorhersage der Mathematikleistung einbezogen. Die Arbeitsgedächtnis- und kognitive Flexibilitätsleistung in den Performanztests waren signifikante Prädiktoren, auch nach Kontrolle des IQ. Die Subskalen der Fremdbeurteilung (Arbeitsgedächtnis, Inhibition und kognitive Flexibilität) klärten keine zusätzliche Varianz auf; die Elternbeurteilung des Arbeitsgedächtnisses verfehlte die Signifikanzgrenze allerdings knapp $(p=.053)$.

In einer klinischen Stichprobe von 8- bis 13-jährigen Kindern klärten sowohl Performanztests zu Inhibition und Arbeitsgedächtnis als auch Eltern- und Lehrkraftrating nach Kontrolle der jeweils anderen Erfassungsmethode einen signifikanten Varianzanteil schulischer Leistungen auf (Soto et al., 2020). Allerdings klärten die Performanztests einen höheren einzigartigen Varianzanteil auf als die Fremdbeurteilungen. Alter, Geschlecht, ADHS- und Medikationsstatus wurden kontrolliert.

Zusammenfassend lässt sich feststellen, dass der prädiktive Wert von EF-Fremdbeurteilungen in einigen Untersuchungen nachgewiesen werden konnte, aber noch nicht hinreichend geklärt ist.

\section{Fragestellung und Hypothesen}

Im Fokus der vorliegenden Untersuchung steht die Frage, ob EF-Elternbeurteilungen zusätzlich zu Performanztests einen signifikanten Vorhersagewert für Grundschulleistungen haben. Spezifischer wird angenommen, dass die Performanztests nach Kontrolle von SÖS, Alter und ADHS-Merkmalen die Mathematik- und Leseleistung von Erst- und Zweitklässler_innen vorhersagen. Bezüglich der Elternbeurteilungen wird angenommen, dass diese darüber hinaus einen zusätzlichen Varianzanteil der Leistungen aufklären. Im Bereich der Rechen- und Leseleistungen wurden das leise Wortdekodieren und die Geschwindigkeit der Addition und Subtraktion im Zahlenraum bis 20 untersucht. Die Rechtschreibleistung konnte aus ökonomischen Gründen nicht mit einbezogen werden. Die Hypothesen werden mit einer Stichprobe geprüft, in der Kinder mit Entwicklungsauffälligkeiten wie ADHS überrepräsentiert sind. 


\section{Methode}

Die Daten entstammen einer 3-jährigen Längsschnittstudie mit insgesamt $N=2084$ - bis 6-jährige Kindern, die jährlich getestet wurden. Die hier verwendeten Variablen wurden hauptsächlich zum dritten Messzeitpunkt erhoben (außer der SÖS). Die EF- Performanztests fanden vormittags in Einzelsitzungen in den Einrichtungen statt; die Schulleistungstests wurden als Gruppentests durchgeführt.

\section{Stichprobe}

Aufgrund von Drop-outs, Ausreißern und fehlenden Werten verblieben $N=96$ Kinder (38\% weiblich; mittleres Alter $M=91$ Monate, $S D=7$ Monate). Davon besuchten $n=$ 54 Kinder die erste Klasse, $n=41$ Kinder die zweite Klasse und $n=1$ Kind eine Kombinationsklasse aus Klassenstufe 1 und 2. Der relativ hohe Drop-out erklärt sich damit, dass alle Kinder mit einem oder mehreren fehlenden Werten (oder Ausreißern) entfernt wurden und keine Imputationen vorgenommen wurden. $N=24$ Kinder zeigten ärztlich diagnostizierte Auffälligkeiten (davon 6 Kinder mit Medikation). Am häufigsten kamen Auffälligkeiten im Bereich Sprachentwicklung $(\mathrm{n}=12)$, motorische Entwicklungsverzögerungen $(n=4)$ und Aufmerksamkeitsstörungen $(n=4)$ vor. Der Rest war nicht näher beschrieben.

\section{Messinstrumente}

\section{Kognitiv-exekutive Tests: Inhibition}

Die Inhibitionsleistung wurde anhand der Go/NoGo-Aufgabe der Arbeitsgedächtnistestbatterie für Kinder von 5 bis 12 Jahren (AGTB 5-12) nach Hasselhorn et al. (2012) erfasst. Die Kinder sollten nur auf eine ganz bestimmte Kriterienkombination mit Tastendruck reagieren („Go“). Abhängige Variable war der Diskriminationsindex A' (Verhältnis richtige zu falschen Reaktionen). Die Retest-Reliabilität ist niedrig $(r=.40)$, ebenso die interne Konsistenz (Cronbach Alpha = .58) (Hasselhorn et al., 2012).

\section{Kognitiv-exekutive Tests: Arbeitsgedächtnis}

Die Arbeitsgedächtniskomponente wurde mittels zweier unterschiedlicher sogenannter Spannenaufgaben erfasst. Bei der Farbspanne rückwärts (AGTB 5-12; Hasselhorn et al., 2012) werden farbige Kreise dargeboten, die anschließend in umgekehrter Reihenfolge auf einem Farbkreis angetippt werden. Abhängige Variable war der Mittelwert der beiden längsten korrekten Spannen. Die Retest-Reliabilität ist niedrig $(r=.49)$, die interne Konsistenz hoch $(r=.96)$ (Hasselhorn et al., 2012).
Die visuell-räumlich dynamische Corsi Blocks rückwärts-Aufgabe wurde in Anlehnung an das Wiener Testsystem (WTS,; Schellig, 2010) durchgeführt. Aus neun unsystematisch angeordneten Holzklötzchen auf einem Brett (für das Kind unsichtbar nummeriert) wurden pro Durchgang 2-7 Klötze von der Versuchsleitung berührt und sollten vom Kind danach in umgekehrter Reihenfolge angetippt werden. Abhängige Variable war die längste korrekt wiedergegebene Spanne. Bei Erwachsenen ist die interne Konsistenz akzeptabel (Cronbach Alpha = .76; Schellig, 2010).

\section{Kognitiv-exekutive Tests: Flexibilität}

Die kognitive Flexibilität wurde mit der gemischten Flanker-Aufgabe (nach Eriksen \& Eriksen, 1974; entwickelt an der Universität Bern, vgl. Michel, Röthlisberger, Neuenschwander \& Roebers, 2011). Am Laptopbildschirm wurden fünf farbige Fische dargeboten. Waren die Fische rot, sollte auf die Blickrichtung des mittleren Fischs mit Tastendruck reagiert werden, bei gelben Fischen auf die der äußeren. Abhängige Variable war die mittlere Reaktionszeit aller 24 Durchgänge.

\section{Elternfragebögen}

Als Elternfragebogen wurde das BRIEF-Verhaltensinven$\operatorname{tar}$ (Drechsler, Steinhausen \& Gioia, 2013) eingesetzt. Dieses umfasst 86 Fragen zu EF-Defiziten in acht Subskalen, aus denen drei Indizes gebildet werden: Der Verhaltensregulations-Index (VRI) (aus den Skalen Hemmen, Umstellen und emotionale Kontrolle), der Kognitive RegulationsIndex (KRI) (aus den Skalen Initiative, Arbeitsgedächtnis, Planen/Strukturieren, Ordnen/Organisieren und Überprüfen) und ein „exekutiver Gesamtwert“. Die interne Konsistenz ist ausgezeichnet (Cronbachs Alpha (KRI) = .98, Cronbachs Alpha (VRI) $=.94)$, die Retest-Reliabilität sehr gut $(r(\mathrm{KRI})=.87, r(\mathrm{VRI})=.83)$ (Drechsler et al., 2013). Die psychometrische Struktur der deutschsprachigen Version (Konstrukt- und Kriteriumsvalidität) konnte von Schöfl, Beitel, Kloo \& Kaufmann (2014) validiert werden.

Ein Richtwert für den SÖS wurde anhand der Ausbildung und beruflichen Situation beider Eltern ermittelt. In Anlehnung an die Magnitude-Prestige-Skala (Wegener, 1988) wurde den Ausbildungsarten und den aktuellen Berufen ein Indexwert von 1 bis 6 zugeordnet. Abhängige Variable war die Summe, wobei der Beruf mit dem höchsten Wert doppelt gewertet wurde. Die Reliabilität ist zufriedenstellend $(r=.78)$ (Wegener, 1988).

Die Erfassung der ADHS-Kernmerkmale erfolgte anhand der 10 Fragen des ADHS-Index der Conners 3-Skalen zu Aufmerksamkeit und Verhalten nach Lidzba, Christiansen und Drechsler (2013). Abhängige Variable war die transformierte Rohwertsumme (nach Manual). Die Sensitivität liegt bei .88, die Spezifität bei .91 (Lidzba et al., 
2013). Zusätzlich wurden Fragen zu ärztlich diagnostizierten Auffälligkeiten und Medikation gestellt.

\section{Schulleistungstests}

Die Schulleistungstests wurden zum Schuljahresende durchgeführt. Die Lesefähigkeit (Lesetempo; Dekodierfähigkeit) der Kinder wurde durch die Würzburger Leise Leseprobe - Revision (WLLP-R; Schneider, Blanke, Faust \& Küspert, 2011) erfasst. In untereinander angeordneten Reihen sind je ein Wort und vier Bilder abgebildet; das korrespondierende Bild soll markiert werden. Abhängige Variable war die Anzahl der in 5 Minuten korrekt gelösten Aufgaben (insgesamt 140). Die Retest-Reliabilität liegt bei $r=.93$ (über die vier Klassenstufen; Schneider et al., 2011).

Die mathematischen Fähigkeiten wurden anhand der Subtests Addition und Subtraktion mit Zehnerübergang im Zahlenraum von 1 bis 20 aus dem Diagnostischen Inventar zu Rechenfertigkeiten im Grundschulalter (DIRG; Grube, Weberschock, Blum \& Hasselhorn, 2010) untersucht. Abhängige Variable war der Mittelwert der Anzahl richtig gelöster Items aus beiden Subtests. Die Retest-Reliabilität ist zufriedenstellend $(r=.71-.84)$ (Grube et al., 2010).

\section{Ergebnisse}

\section{Deskriptive Statistiken}

Tabelle 1 zeigt die deskriptiven Statistiken der einbezogenen Variablen. Insgesamt ist nicht von Boden- oder Deckeneffekten auszugehen.

Die durchschnittlichen T-Werte (berechnet wenn möglich) liegen im Normalbereich. Insgesamt zeigten $26 \%$ der Kinder ein erhöhtes ADHS-Risiko, $20 \%$ eine unterdurchschnittliche Mathematikleistung, $11 \%$ eine unterdurchschnittliche Leseleistung und 6\% unterdurchschnittliche Arbeitsgedächtnisleistungen (Farbspanne rückwärts). Im BRIEF zeigten $10 \%$ der Kinder im VerhaltensregulationsIndex Auffälligkeiten, 5\% im Kognitiven Regulations-Index.

Tabelle 1. Deskriptive Statistiken der einbezogenen Variablen

\begin{tabular}{|c|c|c|c|}
\hline & $M$ & $S D$ & Min-Max \\
\hline Alter (Monate) & 91.08 & 7.31 & $76-106$ \\
\hline SÖS (Rohwert) & 16.58 & 5.47 & $7-30$ \\
\hline $\begin{array}{l}\text { ADHS-Merkmale (Conners 3: Transformierte } \\
\text { Rohwertsumme) }\end{array}$ & 3.30 & 3.75 & $0-13$ \\
\hline ADHS-Merkmale (Conners 3: T-Wert) & 55.99 & 6.08 & $50-73$ \\
\hline Corsi Blocks rückwärts (längste relevante Spanne) & 3.91 & 1.10 & $2-7$ \\
\hline $\begin{array}{l}\text { Farbspanne rückwärts (Mittelwert der beiden } \\
\text { längsten korrekt wiedergegebenen Spannen) }\end{array}$ & 3.14 & 0.74 & $2-5$ \\
\hline Farbspanne rückwärts (T-Wert) & 52.48 & 8.41 & $36-71$ \\
\hline Go / NoGo (Diskriminationsindex Á) & .86 & .09 & $.54-.98$ \\
\hline Gemischte Flanker (Reaktionszeit in ms) & 1563 & 481 & $723-3179$ \\
\hline Verhaltensregulations-Index (BRIEF: Rohwert) & 41.22 & 10.31 & $28-77$ \\
\hline Verhaltensregulations-Index (BRIEF: T-Wert) & 49.85 & 9.52 & $37-82$ \\
\hline Kognitiver Regulations-Index (BRIEF: Rohwert) & 68.35 & 14.15 & $45-114$ \\
\hline Kognitiver Regulations-Index (BRIEF: T-Wert) & 50.21 & 8.30 & $37-79$ \\
\hline Leseleistung (WLLP-R: Rohwert) & 55.29 & 21.25 & $4-101$ \\
\hline Leseleistung (WLLP-R:T-Wert) & 52.01 & 9.97 & $25-71$ \\
\hline $\begin{array}{l}\text { Mathematikleistung (DIRG: Mittelwert aus den } \\
\text { Rohwerten der Subtests Addition und Subtraktion) }\end{array}$ & 24.36 & 16.14 & $1-70.5$ \\
\hline $\begin{array}{l}\text { Mathematikleistung (DIRG: Mittelwert aus den } \\
\text { T-Werten der Subtests Addition und Subtraktion) }\end{array}$ & 48.28 & 9.92 & $24-71.5$ \\
\hline
\end{tabular}

Anmerkungen: BRIEF = Behavior Rating Inventory of Executive Function; WLLP-R = Würzburger Leise Leseprobe - Revision; DIRG = Diagnostisches Inventar zu Rechenfertigkeiten im Grundschulalter. 


\section{Voranalysen}

Geschlechtsunterschiede in allen Variablen wurden anhand von zweiseitigen t-Tests geprüft. Lediglich beim Alter zeigte sich ein Unterschied, $t(94)=-2.33 ; p=.022, d=$ 0.49: Die Jungen waren im Schnitt dreieinhalb Monate älter als die Mädchen.

Tabelle 2 zeigt die Pearson-Korrelationen (unterhalb der Diagonalen alterskontrollierte Partialkorrelationen).

Nach Alterskontrolle zeigte sich ein niedriger signifikanter Zusammenhang zwischen Arbeitsgedächtnis- (Farbspanne rückwärts) und Inhibitionsleistung (Go/NoGo). Aufgrund der niedrigen Aufgabeninterkorrelationen wurde auf die Bildung einer aggregierten EF-Variable verzichtet. Bzgl. Performanztests und Elternbeurteilungen korrelierten nach Kontrolle des Alters lediglich kognitive Flexibilität und Verhaltensregulations-Index niedrig. Der Verhaltensregulations-Index korrelierte nach Kontrolle des Alters moderat mit den ADHS-Merkmalen, der Kognitive Regulations-Index hoch.

Alle vier kognitiv-exekutiven Testverfahren korrelierten nach der Kontrolle des Alters niedrig bis moderat, größtenteils signifikant, mit den Mathematik- und Leseleistungen. Bzgl. Elternbeurteilung korrelierte lediglich der Kognitive Regulations-Index nach Alterskontrolle niedrig mit der Leseleistung.

\section{Vorhersage der Leseleistung}

Residuenplots, Linearität, Homoskedastizität, Multikollinearität und Autokorrelation der Residuen wurde geprüft; die Voraussetzungen können nach Entfernung von drei Ausreißerwerten als erfüllt gelten. Die Ergebnisse der hier- archischen multiplen Regressionen zur Vorhersage der Lese- und Mathematikleistung sind in Tabelle 3 dargestellt.

Im ersten Schritt erklärten SÖS, Alter und ADHS-Symptome gemeinsam $17 \%$ der Varianz der Leseleistung, $F(3,90)=7.50, p<.001$. SÖS $[\beta=.22, t(90)=2.30, p=.024]$ und Alter $[\beta=.41, t(90)=4.34, p<.001]$ waren signifikante Einzelprädiktoren. In einem zweiten Schritt klärten die kognitiv-exekutiven Testverfahren weitere $25 \%$ Varianz auf, $F(4,86)=10.40, p<.001$. Von den exekutiven Testverfahren erwiesen sich Inhibition $[\beta=.23, t(86)=2.70, p=$ $.008]$, Arbeitsgedächtnis (Farbspanne rückwärts) $[\beta=.35$, $t(86)=3.82, p<.001]$ und kognitive Flexibilität $[\beta=-.24$, $\mathrm{t}(86)-2.83, p=.006]$ als signifikante Einzelprädiktoren. In einem dritten Schritt klärten die verhaltensbezogenen EFElternbeurteilungen zusätzlich einen signifikanten Varianzanteil auf, $F(2,84)=3,62, p=.031, \Delta R^{2}=.03$. Ausschließlich der Kognitive Regulations-Index war signifikanter Einzelprädiktor $[\beta=-.34, t(84)=-2.69, p=.009)]$. Auch nach Hinzunahme der Elternbeurteilungen blieben die Performanztests zu Inhibition $[\beta=.22, t(84)=2.62, p=.010)]$, Arbeitsgedächtnis $[\beta=.30, t(84)=3.30, p=.001)]$ und kognitiver Flexibilität $[\beta=-.28, t(84)=-3.25, p=.002)]$ signifikante Einzelprädiktoren. Gemeinsam klärten die beiden EF-Erfassungsmethoden einen Varianzanteil von $28 \%$ in der Leseleistung auf, was einem großen Effekt $\left(f^{2}=0.39\right)$ entspricht (Cohen, 1992).

\section{Vorhersage der Mathematikleistung}

SÖS, Alter und ADHS-Symptome erklärten insgesamt $15 \%$ der Varianz in der Mathematikleistung, $F(3,89)=$ $6.47, p<.001$. Ausschließlich das Alter $[\beta=.38, t(89)=$ 3.96, $p<.001]$ war signifikanter Einzelprädiktor. In

Tabelle 2. Pearson-Korrelationen der einbezogenen Variablen. Alterskontrollierte Partialkorrelationen unter der Diagonalen

\begin{tabular}{|c|c|c|c|c|c|c|c|c|c|c|}
\hline & 1 & 2 & 3 & 4 & 5 & 6 & 7 & 8 & 9 & 10 \\
\hline 1 Sozioökomischer Status & & -.10 & $.21 *$ & .14 & $.29 * \star$ & .04 & -.13 & -.10 & .19 & .16 \\
\hline 2 ADHS-Merkmale & -.09 & & $.21 *$ & -.16 & -.00 & $.21 *$ & $.49 * *$ & $.59 * \star$ & -.03 & -.12 \\
\hline 3 Go / NoGo & $.22^{*}$ & $.21 *$ & & .17 & $.23 *$ & -.07 & .13 & .11 & $.34 * *$ & $.21 *$ \\
\hline 4 Corsi Blocks rückwärts & .18 & -.18 & .16 & & $.25^{\star}$ & $-.24 *$ & -.00 & .00 & $.26 *$ & $.31 * *$ \\
\hline 5 Farbspanne rückwärts & $.34 * \star$ & -.02 & $.22^{\star}$ & .20 & & -.17 & .04 & -.09 & $.45^{\star \star}$ & $.40 * \star$ \\
\hline 6 Gemischte Flanker & .01 & $.22^{\star}$ & -.06 & -.20 & -.12 & & $.23 *$ & .13 & $-.38 * \star$ & $-.29 * *$ \\
\hline 7 Verhaltensregulations-Index & -.13 & $.49 * *$ & .13 & -.02 & .03 & $.25^{\star}$ & & $.72 * \star$ & -.03 & .05 \\
\hline 8 Kognitiver Regulations-Index & -.11 & $.59 * *$ & .11 & .01 & -.09 & .13 & $.72^{\star \star}$ & & -.20 & -.13 \\
\hline 9 Leseleistung & $.24^{*}$ & -.06 & $.33 * *$ & .18 & $.40 * \star$ & $-.34 * \star$ & -.05 & $-.20^{*}$ & & $.58^{\star *}$ \\
\hline 10 Mathematikleistung & $.22 *$ & -.15 & .19 & $.24 *$ & $.33 * \star$ & $-.23^{\star}$ & .03 & -.13 & $.51 * \star$ & \\
\hline
\end{tabular}

Anmerkungen: $* p<.05 ; * * p<.01$ 
einem zweiten Schritt klärten die Performanztests weitere $9 \%$ der Varianz auf, $F(4,85)=3.53, p=.010$. Nur die Arbeitsgedächtnisleistung (Farbspanne rückwärts) war hierbei signifikanter Einzelprädiktor, $\beta=.25, t(85)=2.48$, $p=.015$. Die Elternbeurteilungen klärten zusätzlich in einem dritten Schritt $4 \%$ Varianz auf, $F(2,83)=3.68, p=$ .030. Sowohl der Kognitive Regulations-Index [ $\beta=-.34$, $t(83)=-2.33, p=.022]$ als auch der Verhaltensregulations-Index $[\beta=.36, t(83)=2.57, p=.012]$ waren signifikante Einzelprädiktoren. Der VerhaltensregulationsIndex zeigte jedoch einen positiven Zusammenhang mit der Mathematikleistung. Die Arbeitsgedächtnisleistung (Farbspanne rückwärts) war auch nach Hinzunahme der BRIEF-Indizes signifikant $[\beta=.20, t(83)=2.00, p=.049]$. Gemeinsam erklärten die beiden EF-Erfassungsmethoden einen Varianzanteil von $13 \%$ in der Mathematikleistung, was einem mittleren Effekt $\left(f^{2}=0.15\right)$ entspricht (Cohen, 1992).

\section{Diskussion}

Ziel der Studie war ein besseres Verständnis der Vorhersagekraft von EF- Performanztests und Elternbeurteilungen für Grundschulleistungen. Nach Kontrolle von SÖS, Alter und ADHS-Merkmalen lieferten sowohl die Performanztests als auch die Elternbeurteilungen einen bedeutsamen Vorhersagewert für die Mathematik- und Leseleistung. Bisherige Befunde zu separater Leistungsvorhersage durch beide Methoden (Locascio et al., 2010; Miranda et al., 2015; Zorrilla-Silvestre et al., 2016) werden durch die Erkenntnis erweitert, dass die gemeinsame Einbeziehung gewinnbringend ist. Elternbeurteilungen konnten inkrementell einen niedrigen, aber bedeutsamen Varianzanteil aufklären. Dies erweitert Befunde, in denen Aussagen über den Varianzanteil, den die beiden EF-Maße gemeinsam aufklären, getroffen werden konnten (Ten Eycke \& Dewey, 2016). Es erscheint vor diesem Befundmuster

Tabelle 3. Hierarchische Regressionen zur Vorhersage der Leseleistung und Mathematikleistung (in Klammern)

\begin{tabular}{|c|c|c|c|c|c|c|c|c|c|}
\hline & \multicolumn{3}{|c|}{ Schritt 1} & \multicolumn{3}{|c|}{ Schritt 2} & \multicolumn{3}{|c|}{ Schritt 3} \\
\hline & $B$ & $S E(B)$ & $\beta$ & $B$ & $S E(B)$ & $\beta$ & $B$ & $S E(B)$ & $\beta$ \\
\hline Konstante & $\begin{array}{r}-66.47 \\
(-54.97)\end{array}$ & $\begin{array}{r}26.26 \\
(19.31)\end{array}$ & & $\begin{array}{r}-67.55 \\
(-53.80)\end{array}$ & $\begin{array}{r}28.49 \\
(23.08)\end{array}$ & & $\begin{array}{r}-40.23 \\
(-40.18)\end{array}$ & $\begin{array}{r}29.91 \\
(24.23)\end{array}$ & \\
\hline Sozioökonomischer Status & $\begin{array}{r}.83 \\
(.50)\end{array}$ & $\begin{array}{r}.36 \\
(.27)\end{array}$ & $\begin{array}{l}.22^{\star} \\
(.18)\end{array}$ & $\begin{array}{r}.18 \\
(.16)\end{array}$ & $\begin{array}{r}.33 \\
(.28)\end{array}$ & $\begin{array}{r}.05 \\
(.06)\end{array}$ & $\begin{array}{r}.24 \\
(.28)\end{array}$ & $\begin{array}{r}.33 \\
(.28)\end{array}$ & $\begin{array}{r}.06 \\
(.10)\end{array}$ \\
\hline Alter & $\begin{array}{l}1.19 \\
(.79)\end{array}$ & $\begin{array}{r}.28 \\
(.20)\end{array}$ & $\begin{array}{c}.41 * \star \star \\
(.38 * \star \star\end{array}$ & $\begin{array}{r}.63 \\
(.50)\end{array}$ & $\begin{array}{r}.26 \\
(.21)\end{array}$ & $\begin{array}{r}.22^{\star} \\
\left(.25^{\star}\right)\end{array}$ & $\begin{array}{r}.56 \\
\left(.46^{\star}\right)\end{array}$ & $\begin{array}{r}.25 \\
(.20)\end{array}$ & $\begin{array}{r}.19 * \\
\left(.22^{\star}\right)\end{array}$ \\
\hline ADHS-Merkmale & $\begin{array}{r}-.10 \\
(-.55)\end{array}$ & $\begin{array}{r}0.52 \\
(-.38)\end{array}$ & $\begin{array}{r}-.02 \\
(-.14)\end{array}$ & $\begin{array}{r}-.06 \\
(-.49)\end{array}$ & $\begin{array}{r}.47 \\
(0.39)\end{array}$ & $\begin{array}{r}-.01 \\
(-.12)\end{array}$ & $\begin{array}{r}.55 \\
(-.32)\end{array}$ & $\begin{array}{r}.57 \\
(.47)\end{array}$ & $\begin{array}{r}.10 \\
(-.08)\end{array}$ \\
\hline Go/NoGo & & & & $\begin{array}{r}52.91 \\
(16.50)\end{array}$ & $\begin{array}{r}19.62 \\
(16.12)\end{array}$ & $\begin{array}{r}.23 * \star \\
(.10)\end{array}$ & $\begin{array}{r}50.07 \\
(13.03)\end{array}$ & $\begin{array}{r}19.10 \\
(15.69)\end{array}$ & $\begin{array}{l}.22^{\star} \\
(.08)\end{array}$ \\
\hline Corsi Blocks rückwärts & & & & $\begin{array}{r}.58 \\
(1.50)\end{array}$ & $\begin{array}{r}1.65 \\
(1.39)\end{array}$ & $\begin{array}{r}.03 \\
(.11)\end{array}$ & $\begin{array}{r}1.13 \\
(1.66)\end{array}$ & $\begin{array}{r}1.63 \\
(1.37)\end{array}$ & $\begin{array}{r}.06 \\
(.12)\end{array}$ \\
\hline Farbspanne rückwärts & & & & $\begin{array}{r}9.87 \\
(5.22)\end{array}$ & $\begin{array}{r}2.58 \\
(2.10)\end{array}$ & $\begin{array}{r}.35 \star \star \star \\
(.25 \star)\end{array}$ & $\begin{array}{r}8.47 \\
(4.16)\end{array}$ & $\begin{array}{r}2.57 \\
(2.08)\end{array}$ & $\begin{array}{l}.30^{\star *} \\
\left(.20^{\star}\right)\end{array}$ \\
\hline Gemischte Flanker & & & & $\begin{array}{l}-.01 \\
(.00)\end{array}$ & $\begin{array}{r}.00 \\
(.00)\end{array}$ & $\begin{array}{r}-.24 * \star \\
(-.13)\end{array}$ & $\begin{array}{r}-.01 \\
(-.01)\end{array}$ & $\begin{array}{l}.00 \\
(.00)\end{array}$ & $\begin{array}{r}-.28^{* *} \\
(-.19)\end{array}$ \\
\hline Verhaltensregulations-Index & & & & & & & $\begin{array}{r}.42 \\
(.52)\end{array}$ & $\begin{array}{r}.24 \\
(.20)\end{array}$ & $\begin{array}{r}.21 \\
\left(.36^{\star}\right)\end{array}$ \\
\hline Kognitiver Regulations-Index & & & & & & & $\begin{array}{r}-.49 \\
(-.36)\end{array}$ & $\begin{array}{r}.18 \\
(.16)\end{array}$ & $\begin{array}{l}-.34^{\star \star} \\
\left(-.34^{\star}\right)\end{array}$ \\
\hline$\Delta R^{2}$ & & & & & $\begin{array}{r}.25^{\star \star \star} \\
(.09 *)\end{array}$ & & & $\begin{array}{c}.03^{\star} \\
\left(.04^{\star}\right)\end{array}$ & \\
\hline Korrigiertes $R^{2}$ & & $\begin{array}{l}.17 \star \star \star \\
\left(.15^{\star \star \star}\right)\end{array}$ & & & $\begin{array}{c}.42^{\star \star \star} \\
\left(.24^{\star \star \star}\right)\end{array}$ & & & $\begin{array}{r}.45^{\star \star \star} \\
(.28 * \star \star\end{array}$ & \\
\hline
\end{tabular}

Anmerkungen: ${ }^{*} p<.05,{ }^{* *} p<.01,{ }^{* * *} p<.001$ 
sinnvoll, beide Verfahren in Kombination einzusetzen, um Risikokinder früh identifizieren und fördern zu können. $\mathrm{Zu}$ einem ähnlichen Schluss kommen auch Schöfl et al. (2015) bzgl. der Erfassung von Planungsleistungen als eine Komponente der EF bei Kindern mit ADHS mittels BRIEF-Elternfragebogen und Performanztest.

Der insgesamt aufgeklärte Varianzanteil der Schulleistungen ist in vorliegender Studie beachtlich $(45 \%$ bei Leseleistungen und $28 \%$ bei Mathematikleistungen). Dass die Performanztests in vorliegender Studie einen höheren Varianzanteil (25\% bzw. $9 \%$ ) als die Elternbeurteilungen aufklären, ist methodisch begründet. Die Kriteriumsvarianz, die beide Erfassungsmethoden teilen, wird den zuerst hinzugefügten Performanztests zugeschrieben.

Ein Vorteil der vorliegenden Studie ist, dass mathematische und Leseleistungen separat betrachtet wurden, während in früheren Studien häufig Gesamtwerte genutzt wurden (Diamantopoulou, Rydell, Thorell \& Bohlin, 2007; Langberg, Dvorsky \& Evans, 2013; Soto et al., 2020; Wirth, Reinelt, Gawrilow \& Rauch, 2015). Der gesamte aufgeklärte Varianzanteil war dabei für die Leseleistungen deutlich höher als für die Mathematikleistungen, wie es auch in früheren Studien der Fall war (Thorell, 2007; Ten Eycke \& Dewey, 2016). Möglicherweise sind EF bei Leseanfänger_innen besonders relevant, da diese im Vergleich zu Fortgeschrittenen mehr Wörter einzeln entschlüsseln müssen und sie noch nicht automatisch aus dem Sichtwortschatz abrufen können (vgl. Marx, 2007; Ober et al., 2020). Bei den mathematischen Fähigkeiten wird hingegen davon ausgegangen, dass die EF-Beteiligung beim Lernen zunehmend komplexer Konzepte mit der Klassenstufe ansteigt (Fuhs, Farran \& Nesbitt, 2015).

Die Annahme, dass die Erfassungsmethoden unterschiedliche Aspekte eines heterogenen EF-Konstrukts erfassen (Barkley \& Fischer, 2011; McAuley et al., 2010; Toplak et al., 2013), wurde auch hier durch die niedrigen Interkorrelationen gestützt. Theoretische Annahmen darüber, dass die beiden EF-Erfassungsmethoden z.B. verschiedene Ebenen einer Zielhierarchie abbilden (Barkley \& Fischer, 2011) oder sich in der Art der Leistungssituation (Toplak et al., 2013) oder emotionalen Beteiligung unterscheiden (z.B. Ritter et al., 2014), scheinen vor dem Hintergrund der vorliegenden Befunde plausibel. In den unterschiedlichen Facetten des „Lernalltags“, die die schulischen Leistungen beeinflussen, scheinen all diese EF-Aspekte relevant zu sein.

Zur Vorhersage der Leseleistung waren sowohl Arbeitsgedächtnisleistung (Farbspanne rückwärts) als auch Inhibition und kognitive Flexibilität bedeutsam, in Einklang mit bestehenden Forschungsergebnissen (Follmer, 2018; Michel et al., 2019; Ober et al., 2020; Roebers et al., 2014). Die Aufgabe Farbspanne rückwärts war dabei ein eher phonologisches Maß, da die Kinder die Farben in der Regel verbal benannten. Visuell-räumlich dynamische Arbeitsgedächtnisleistungen (Corsi Blocks rückwärts) waren hingegen wie auch in früheren Studien nicht prädiktiv (Miranda et al., 2015; Brandenburg et al., 2013). Einige Forschergruppen nehmen an, dass das visuell-räumliche Arbeitsgedächtnis spezifisch für die Vorhersage der Mathematikleistung ist (Bull et al., 2008). Dies war in vorliegender Studie nicht der Fall. Hier könnte die Aufgabenart eine Rolle spielen (vgl. Best et al. 2011): Visuell-räumliche Aspekte scheinen vor allem für die Einschätzung von Größen symbolischer Zahlen (Simmons, Willis \& Adams, 2012) oder die Manipulation und Zuordnung von Größen auf dem Zahlenstrahl relevant (Bull et al., 2008), die in der vorliegenden Untersuchung nicht verwendet wurden.

Für die Vorhersage der Mathematikleistung war von den EF-Performanztests ausschließlich die Arbeitsgedächtnisleistung (Farbspanne rückwärts) bedeutsam. Dies steht im Einklang mit früheren Befunden zur besonderen Rolle des Arbeitsgedächtnisses für mathematische Kompetenzen (Bull \& Lee, 2014; Van der Ven et al., 2012; Visu-Petra et al., 2011). Interessant ist, dass alle vier exekutiven Testverfahren signifikant mit der Mathematikleistung korreliert waren, im Regressionsmodell aber nur die Farbspanne rückwärts signifikant wurde. Dies passt zu den Befunden von Van der Ven et al. (2012), bei denen Arbeitsgedächtnis und ein gemeinsamer Inhibition / Shifting-Faktor bei separater Betrachtung einen signifikanten Vorhersagewert für die Mathematikleistung (7-8 Jahre) lieferten, bei einer gemeinsamen Einbeziehung jedoch nur der Arbeitsgedächtnisfaktor prädiktiv war. Möglicherweise sind Arbeitsgedächtnisfähigkeiten für die Aufrechterhaltung von Inhalten wichtig, die für das erfolgreiche Bewältigen von kognitive Flexibilitäts- und Inhibitionsaufgaben notwendig sind (Bull \& Lee, 2014; Garon, Bryson \& Smith, 2008).

Die mittelhohen, meist signifikanten Zusammenhänge der EF-Performanztests mit beiden Leistungsdomänen passen zur Befundlage (z.B. Gerst et al., 2017; Jacobson, Williford \& Pianta, 2011; Roebers et al., 2014; Soto et al., 2020). Interessanterweise sind die Korrelationen der EFPerformanztests mit den Schulleistungstests höher als mit den Elternbeurteilungen, die dasselbe Konstrukt messen sollten.

Bezüglich der Elternbeurteilungen erwies sich der Kognitive Regulations-Index als signifikant zur Vorhersage der Leseleistung und beide Indizes als signifikant zur Vorhersage der Mathematikleistung. Widersprüchlich erschien jedoch, dass höhere Defizite in der Verhaltensregulation mit einer besseren Mathematikleistung verknüpft waren. Dies zeigte sich auch in anderen Untersuchungen (Miranda et al., 2015; Ten Eycke \& Dewey, 2016; Zorrilla-Silvestre et al., 2016). Eine Erklärung könnten die hohen Interkorrelationen zwischen den beiden BRIEF-Indizes sein (Dekker et al., 2017; Drechsler et al., 2013; Gerst et al., 2017; Lang- 
berg et al., 2013). Auch wenn in den durchgeführten Regressionen nicht von Multikollinearität auszugehen war, können hoch korrelierte Prädiktoren mit einem hohen Anteil an geteilter Kriteriumsvarianz einhergehen (Tabachnick \& Fidell, 2014). Bei einer Betrachtung der Korrelationen zwischen dem Verhaltensregulations-Index und der Mathematikleistung fällt auf, dass diese nahe Null liegen. Erst in Kombination mit anderen Prädiktoren erwies sich der Verhaltensregulations-Index als signifikant. Daher sollte dieser Befund mit Vorsicht interpretiert werden. Insgesamt scheint der Kognitive Regulations-Index besser zur Vorhersage schulischer Leistungen geeignet zu sein als der Verhaltensregulations-Index (Colomer, Berenguer, Roselló, Baixauli \& Miranda, 2017; Di Pinto, 2006; Langberg et al., 2013; Miranda et al., 2015).

\section{Limitationen}

Etwa ein Viertel der Gesamtstichprobe zeigte Entwicklungsauffälligkeiten, die häufig mit exekutiven Problemen einhergehen; dadurch könnten die Zusammenhänge überschätzt werden (Kiese-Himmel, 2020). Dennoch stellt sich in der Praxis die Frage der EF-Diagnostik häufig bei Kindern mit Auffälligkeiten, weswegen insbesondere die Einbeziehung dieser Populationen wichtig ist (vgl. Soto et al., 2020). So deuten z.B. einige Studien darauf hin, dass $\mathrm{Zu}$ sammenhänge zwischen ADHS-Symptomen und unterdurchschnittlichen Schulleistungen teilweise durch EFDefizite, v. a. im Bereich Arbeitsgedächtnis, erklärt werden können (z. B. Biederman et al., 2004; Rennie, Beebe-Frankenberger \& Swanson, 2014; Rogers, Hwang, Toplak, Weiss \& Tannock, 2011).

Ein anderer Kritikpunkt ist, dass wichtige weitere Prädiktoren für schulische Leistungen nicht einbezogen werden konnten, insbesondere bereichsspezifische wie die phonologische Bewusstheit, Wortschatz, Zählfähigkeit oder Mengenbewusstsein (Geary, Hoard, Byrd-Craven, Nugent \& Numtee, 2007; Jun Zhang \& Bin Anual, 2008; Metz, Fröhlich, Rißling \& Petermann, 2011), aber auch weitere domänunspezifische wie Intelligenz, familiäre Lernumwelt und motivationale Faktoren (Kriegbaum, Becker \& Spinath, 2018; Niklas, 2015). Zukünftige Studien sollten diese kontrollieren.

Ferner wurden aufgrund der Stichprobengröße die Daten der Erst- und Zweitklässler_innen gemeinsam in die Analysen einbezogen. Bei der Interpretation der Befunde sollte bedacht werden, dass die erfassten Rechen- und Leseleistungen möglicherweise auf unterschiedlichen Fertigkeiten beruhen. So sind leises Dekodieren und Addition und Subtraktion bei Zweitklässler_innen evtl. automatisierter als bei Erstklässler_innen, was sich in unterschiedlicher Beteiligung der EF widerspiegeln könnte.

\section{Relevanz für die Praxis}

Eine gemeinsame Einbeziehung von Performanztests und Elternbeurteilungen der EF zur Vorhersage schulischer Leistungen ist sinnvoll; (elternbeurteilte) ADHS-Symptome scheinen dagegen nicht prognostisch relevant.

Ferner legen die Ergebnisse nahe, dass eine EF-Förderung im frühen Grundschulalter positive Effekte auf die schulischen Leistungen haben sollte. Lehrkräfte äußern Bedarf nach Fortbildungen zur Erkennung exekutiver Defizite und Förderung im Unterricht (Dunlap, 2020); dies wäre ein wichtiger Ansatzpunkt. Einige bestehende Förderprogramme scheinen zu einer Verbesserung der EF zu führen, jedoch sind Transfereffekte auf schulische Leistungen nicht konsistent zu beobachten (Bigorra, Garolera, Guijarro \& Hervás, 2016; Goldin et al., 2014; Melby-Lervåg \& Hulme, 2013; Sala \& Gobet, 2017; Titz \& Karbach, 2014). Das Einbeziehen von Übungen zur Nutzung von EF in (Schul-)alltagssituationen könnte die Wirksamkeit verbessern (vgl. Takacs \& Kassai, 2019).

\section{Literatur}

Bailey, C. E. (2007). Cognitive accuracy and intelligent executive function in the brain and in business. Annals of the New York Academy of Sciences, 1118,122 - 141. http://dx.doi.org/10.1196/ annals.1412.011

Banich, M. (2009). Executive Function: The search for an integrated account. Current Directions in Psychological Science 18(2), 89-94. http://dx.doi.org/10.1111/j.1467-8721.2009.01615.x

Barkley, R. A. \& Fischer, M. (2011). Predicting impairment in major life activities and occupational functioning in hyperactive children as adults: Self-reported executive function (EF) deficits versus EF tests. Developmental Neuropsychology, 36(2), 137 - 161. http://dx.doi.org/10.1080/87565641.2010.549877

Best, J. R., Miller, P. H. \& Naglieri, J. A. (2011). Relations between executive function and academic achievement from ages 5 to 17 in a large, representative national sample. Learning and Individual Differences, 21(4), 327 - 336. http://dx.doi.org/10.1016/j. lindif.2011.01.007

Biederman, J., Monuteaux, M., Doyle, A., Seidman, L., Wilens, T., Ferrero et al. (2004). Impact of executive function deficits and attention-deficit/hyperactivity disorder (ADHD) on academic outcomes in children. Journal of Consulting and Clinical Psychology, 72(5), 757 - 766. doi:http://dx.doi.org/10.1037/0022-006X. 72.5.757

Bigorra, A., Garolera, M., Guijarro, S. \& Hervás, A. (2016). Long-term far-transfer effects of working memory training in children with ADHD: A randomized controlled trial. European Child \& Adolescent Psychiatry, 25(8), 853-867. http://dx.doi.org/10.1007/ s00787-015-0804-3

Brandenburg, J., Klesczewski, J., Fischbach, A., Büttner, G., Grube, D., Mähler, C. et al. (2013). Arbeitsgedächtnisfunktionen von Kindern mit Minderleistungen in der Schriftsprache. Lernen und Lernstörungen, 2, 147-159. http://dx.doi.org/10.1024/22350977/a000037

Bull, R., Espy, K. A. \& Wiebe, S. A. (2008). Short-term memory, working memory, and executive functioning in preschoolers: Longitu- 
dinal predictors of mathematical achievement at age 7 years. Developmental Neuropsychology, 33(3), 205 - 228. http://dx.doi. org/10.1080/87565640801982312

Bull, R. \& Lee, K. (2014). Executive functioning and mathematics achievement. Child Development Perspectives, 8(1), 36-41. http://dx.doi.org/10.1111/cdep.12059

Butterfuss, R. \& Kendeou, P. (2018). The role of executive functions in reading comprehension. Educational Psychology Review, 30(3), 801 - 826. http://dx.doi.org/10.1007/s10648-017-9422-6

Clements, D. H., Sarama, J. \& Germeroth, C. (2016). Learning executive function and early mathematics: Directions of causal relations. Early Childhood Research Quarterly, 36, 79-90. http:// dx.doi.org/10.1016/j.ecresq.2015.12.009

Cohen, J. (1992). A power primer. Psychological Bulletin, 112(1), 155 - 159. http://dx.doi.org/10.1037/0033-2909.112.1.155

Colomer, C., Berenguer, C., Roselló, B., Baixauli, I. \& Miranda, A. (2017). The impact of inattention, hyperactivity/impulsivity symptoms, and executive functions on learning behaviors of children with ADHD. Frontiers in Psychology, 8, 1-10. http://dx. doi.org/10.3389/fpsyg.2017.00540

Coltheart, M., Rastle, K., Perry, C., Langdon, R. \& Ziegler, J. (2001). DRC: A dual route cascaded model of visual word recognition and reading aloud. Psychological Review, 108(1), 204-256. http://dx.doi.org/10.1037/0033-295X.108.1.204

Dekker, M. C., Ziermans, T. B., Spruijt, A. M. \& Swaab, H. (2017). Cognitive, parent and teacher rating measures of executive functioning: Shared and unique influences on school achievement. Frontiers in Psychology, 8, 13. http://dx.doi.org/10.3389/fpsyg. 2017.00048

Di Pinto, M. (2006). The ecological validity of the Behavior Rating Inventory of Executive Function (BRIEF) in attention deficit hyperactivity disorder: Predicting academic achievement and social adaptive behavior in the subtypes of ADHD. Philadelphia, Pennsylvania: Drexel University.https://idea.library.drexel.edu/ islandora/object/idea\%3A845/datastream/OBJ/view

Diamantopoulou, S., Rydell, A.-M., Thorell, L. B. \& Bohlin, G. (2007). Impact of executive functioning and symptoms of attention deficit hyperactivity disorder on children's peer relations and school performance. Developmental Neuropsychology, 32(1), 521 - 542. http://dx.doi.org/10.1080/87565640701360981

Diamond, A. (2013). Executive functions. The Annual Review of Psychology, 64, 135-168. http://dx.doi.org/10.1146/annurevpsych-113011-143750

Drechsler, R. (2007). Exekutive Funktionen: Übersicht und Taxonomie. Zeitschrift für Neuropsychologie, 18(3), 233 -248. http:// dx.doi.org/10.1024/1016-264X.18.3.233

Drechsler, R., Steinhausen, H.-C. \& Gioia, G. A. (2013). Verhaltensinventar zur Beurteilung exekutiver Funktionen. Deutschsprachige Adaptation des Behavior Rating Inventory of Executive Function (BRIEF). Bern: Huber.

Dunlap, S. L. (2020). Teacher perceptions about teaching students with executive function deficits., Minneapolis, Minnesota: Walden University.https://scholarworks.waldenu.edu/dissertations 18278

Eriksen, B. A. \& Eriksen, C. W. (1974). Effects of noise letters upon the identification of a target letter in a nonsearch task. Perception \& Psychophysics, 16(1), 143-149. http://dx.doi.org/10. 3758/BF03203267

Follmer, D. J. (2018). Executive function and reading comprehension: A meta-analytic review. Educational Psychologist, 53(1), 42 - 60. http://dx.doi.org/10.1080/00461520.2017.1309295

Fuhs, M. W., Farran, D. C. \& Nesbitt, K. T. (2015). Prekindergarten children's executive functioning skills and achievement gains: The utility of direct assessments and teacher ratings. Journal of Educational Psychology, 107(1), 207-221. http://dx.doi. org/10.1037/a0037366
Garon, N., Bryson, S. \& Smith, I. (2008). Executive function in preschoolers: A review using an integrative framework. Psychological Bulletin, 134, 31-60. http://dx.doi.org/10.1037/00332909.134.1.31

Gathercole, S. \& Alloway, T. (2016). Arbeitsgedächtnis verstehen Ein Leitfaden fürs Klassenzimmer. In S. Kubesch (Hrsg.), Exekutive Funktionen und Selbstregulation: Neurowissenschaftliche Grundlagen und Transfer in die pädagogische Praxis (S.323-336). Bern: Hogrefe

Geary, D. C., Hoard, M. K., Byrd-Craven, J., Nugent, L. \& Numtee, C. (2007). Cognitive mechanisms underlying achievement deficits in children with mathematical learning disability. Child Development, 78(4),1343 - 1359. http://dx.doi.org/10.1111/j.1467-8624. 2007.01069.x

Gerst, E. H., Cirino, P. T., Fletcher, J. M. \& Yoshida, H. (2017). Cognitive and behavioral rating measures of executive function as predictors of academic outcomes in children. Child Neuropsychology: A Journal on Normal and Abnormal Development in Childhood and Adolescence, 23(4), 381 - 407. http://dx.doi.org/1 $0.1080 / 09297049.2015 .1120860$

Gioia, G. A., Isquith, P. K., Guy, S. C. \& Kenworthy, L. (1998). BRIEF: Behavior Rating Inventory of Executive Function. Lutz, FL: Psychological Assessment Resources.

Gioia, G. A., Isquith, P. K., Guy, S. C. \& Kenworthy, L. (2000). BRIEF: Behavior Rating Inventory of Executive Function. Lutz, FL: Psychological Assessment Resources.

Gioia, G. A., Isquith, P. K., Retzlaff, P. D. \& Espy, K. A. (2002). Confirmatory factor analysis of the Behavior Rating Inventory of Executive Function (BRIEF) in a clinical sample. Child Neuropsychology, 8(4), 249-257. http://dx.doi.org/10.1076/chin.8.4. 249.13513

Goldin, A. P., Hermida, M. J., Shalom, D. E., Elias Costa, M., LopezRosenfeld, M., Segretin, M. S. et al. (2014). Far transfer to language and math of a short software-based gaming intervention. Proceedings of the National Academy of Sciences of the United States of America, 111(17), 6443-6448. http://dx.doi.org/10. 1073/pnas.1320217111

Grube, D., Weberschock, U., Blum, M. \& Hasselhorn, M. (2010). Diagnostisches Inventar zu Rechenfertigkeiten im Grundschulalter. Göttingen: Hogrefe.

Hasselhorn, M., Schumann-Hengsteler, R., Gronauer, J., Grube, D., Mähler, C., Schmid, I. et al. (2012). Arbeitsgedächtnistestbatterie für Kinder von 5 bis 12 Jahren:AGTB 5-12 [Working memory test battery for 5- to 12-year-old children]. Göttingen: Hogrefe.

Isquith, P. K., Roth, R. M. \& Gioia, G. A. (2013). Contribution of rating scales to the assessment of executive functions. Applied Neuropsychology: Child, 2(2), 125 -132. http://dx.doi.org/10.1080/216 22965.2013.748389

Jacobson, L. A., Williford, A. P. \& Pianta, R. C. (2011). The role of executive function in children's competent adjustment to middle school. Child Neuropsychology, 17(3), 255 - 280. http://dx.doi.org /10.1080/09297049.2010.535654

Jun Zhang, L. \& Bin Anual, S. (2008). The role of vocabulary in reading comprehension: The case of secondary school students learning English in Singapore. RELC Journal, 39(1), 51-76. http://dx.doi.org/10.1177/0033688208091140

Kiese-Himmel, C. (2020). Das Arbeitsgedächtnis - eine Bestandsaufnahme. Sprache · Stimme · Gehör, 44, 107 -115. http://dx doi.org/10.1055/a-0899-6784

Kriegbaum, K., Becker, N. \& Spinath, B. (2018). The relative importance of intelligence and motivation as predictors of school achievement: A meta-analysis. Educational Research Review, 25, 120-148. https://doi.org/10.1016/j.edurev.2018.10.001

Kubesch, S., Emrich, A. \& Beck, F. (2011). Exekutive Funktionen im Sportunterricht fördern. Sportunterricht, 60(10), 312-316. 
Langberg, J. M., Dvorsky, M. R. \& Evans, S. W. (2013). What specific facets of executive function are associated with academic functioning in youth with attention-deficit/ hyperactivity disorder? Journal of Abnormal Child Psychology, 41(7), 1145-1159. http://dx.doi.org/10.1007/s10802-013-9750-z

Lee, K., Ng, S. F., Pe, M. L., Ang, S. Y., Hasshim, M. N. A. M. \& Bull, R. (2012). The cognitive underpinnings of emerging mathematical skills: Executive functioning, patterns, numeracy, and arithmetic. British Journal of Educational Psychology, 82(1), 82-99. http://dx.doi.org/10.1111/j.2044-8279.2010.02016.x

Lehto, J. E., Juujärvi, P., Kooistra, L. \& Pulkkinen, L. (2003). Dimensions of executive functioning: Evidence from children. British Journal of Developmental Psychology, 21(1), 59-80. https:// psycnet.apa.org/doi/10.1348/026151003321164627

Lidzba, K., Christiansen, H. \& Drechsler, R. (2013). Conners Skalen zu Aufmerksamkeit und Verhalten 3 - Deutschsprachige Adaptation der Conners 3rd Edition von C. Keith Conners. Bern: Hogrefe.

Locascio, G., Mahone, E. M., Eason, S. H. \& Cutting, L. E. (2010). Executive dysfunction among children with reading comprehension deficits. Journal of Learning Disabilities, 43(5), 441 - 454. http://dx.doi.org/10.1177/0022219409355476

Marx, P. (2007). Lese- und Rechtschreiberwerb. Weinheim: Schöningh UTB.

Massol, S., Molinaro, N. \& Carreiras, M. (2015). Lexical inhibition of neighbors during visual word recognition: An unmasked priming investigation. Brain Research, 1604, 35-51. https://doi.org/ 10.1016/j.brainres.2015.01.051

McAuley, T., Chen, S., Goos, L., Schachar, R. \& Crosbie, J. (2010). Is the BRIEF more strongly associated with measures of impairment or executive function? Journal of the International Neuropsychological Society : JINS, 16, 495-505. http://dx.doi.org/ 10.1017/S1355617710000093

Melby-Lervåg, M. \& Hulme, C. (2013). Is working memory training effective? A meta-analytic review. Developmental Psychology, 49(2), 270 - 291. http://dx.doi.org/10.1037/a0028228

Metz, D., Fröhlich, L. P., Rißling, J.-K. \& Petermann, F. (2011). Kurzund Langzeiteffekte einer Förderung der phonologischen Bewusstheit bei Schulanfängern. Zeitschrift für Psychiatrie, Psychologie und Psychotherapie, 59(1), 65-72. http://dx.doi.org/10. 1024/1661-4747/a000053

Michel, E., Molitor, S. \& Schneider, W. (2019). Motor coordination and executive functions as early predictors of reading and spelling acquisition. Developmental Neuropsychology, 44, 1 - 14. http://dx.doi.org/10.1080/87565641.2019.1584802

Michel, E., Röthlisberger, M., Neuenschwander, R. \& Roebers, C. (2011). Development of cognitive skills in children with motor coordination ompairments at 12-month follow-up. Child Neuropsychology: A Journal on Normal and Abnormal Development in Childhood and Adolescence, 17,151 - 172. http://dx.doi.org/10.1 080/09297049.2010.525501

Miranda, A., Colomer, C., Mercader, J., Fernández, M. I. \& Presentación, M. J. (2015). Performance-based tests versus behavioral ratings in the assessment of executive functioning in preschoolers: Associations with ADHD symptoms and reading achievement. Frontiers in Psychology, 6, 545. http://dx.doi. org/10.3389/fpsyg.2015.00545

Miyake, A. \& Friedman, N. P. (2012). The nature and organization of individual differences in executive functions: Four general conclusions. Current Directions in Psychological Science, 21(1), 8 - 14. http://dx.doi.org/10.1177/0963721411429458

Miyake, A., Friedman, N. P., Emerson, M.J., Witzki, A. H., Howerter, A. \& Wager, T. D. (2000). The unity and diversity of executive functions and their contributions to complex "frontal lobe" tasks: A latent variable analysis. Cognitive Psychology, 41(1), 49-100. https://doi.org/10.1006/cogp.1999.0734
Niklas, F. (2015). Die familiäre Lernumwelt und ihre Bedeutung für die kindliche Kompetenzentwicklung. Psychologie in Erziehung und Unterricht, 62, 106-120. http://dx.doi.org/10.2378/peu 2015.art11d

Ober, T. M., Brooks, P.J., Homer, B. D. \& Rindskopf, D. (2020). Executive functions and decoding in children and adolescents: A meta-analytic investigation. Educational Psychology Review, 735-763. http://dx.doi.org/10.1007/s10648-020-09526-0

Otero, T., Barker, L. \& Naglieri, J. (2014). Executive function treatment and intervention in schools. Applied Neuropsychology: Child, 3, 205 - 214. http://dx.doi.org/10.1080/21622965.2014.8 97903

Rennie, B., Beebe-Frankenberger, M., \& Swanson, H. L. (2014). A longitudinal study of neuropsychological functioning and academic achievement in children with and without signs of attention-deficit/hyperactivity disorder. Journal of Clinical and Experimental Neuropsychology, 36(6), 621 -635. doi:http://dx.doi. org/10.1080/13803395.2014.921284

Reynolds, R. E. (2000). Attentional resource emancipation: Toward understanding the interaction of word identification and comprehension processes in reading. Scientific Studies of Reading, 4(3), 169 - 195. http://dx.doi.org/10.1207/S1532799XSSR0403_1

Ritter, B. C., Perrig, W., Steinlin, M. \& Everts, R. (2014). Cognitive and behavioral aspects of executive functions in children born very preterm. Child Neuropsychology, 20(2), $129-144$. http://dx.doi. org/10.1080/09297049.2013.773968

Roebers, C., Röthlisberger, M., Neuenschwander, R., Cimeli, P., Michel, E. \& Jäger, K. (2014). The relation between cognitive and motor performance and their relevance for children's transition to school: A latent variable approach. Human Movement Science, 33, 284 - 297. https://doi.org/10.1016/j.humov.2013.08.011

Rogers, M., Hwang, H., Toplak, M., Weiss, M., \& Tannock, R. (2011). Inattention, working memory, and academic achievement in adolescents referred for attention deficit/hyperactivity disorder (ADHD). Child Neuropsychology, 17(5), 444-458. doi:http:// dx.doi.org/10.1080/09297049.2010.544648

Sala, G. \& Gobet, F. (2017). Working memory training in typically developing children: A meta-analysis of the available evidence. Developmental Psychology, 53(4), 671-685. http://dx.doi.org/ 10.1037/dev0000265

Schellig, D. (2010). Wiener Testsystem Manual Corsi. Mödling: Schuhfried.

Schneider, W., Blanke, I., Faust, V. \& Küspert, P. (2011). Würzburger Leise Leseprobe-Revision (WLLP-R). Ein Gruppentest für die Grundschule (revidierte Version) [Würzburg silent reading test. A group test for elementary school]. Göttingen: Hogrefe.

Schöfl, M., Beitel, C., Kloo, D. \& Kaufmann, L. (2014). Konstrukt-und Kriteriumsvalidität einer deutschen Version des Behavior Rating Inventory of Executive Function (BRIEF) zur Identifikation von Kindern mit Aufmerksamkeitsdefizit-/Hyperaktivitätsstörungen (ADHS). Diagnostica, 60(4), 181-196.

Schöfl, M., Kloo, D. \& Kaufmann, L. (2015). Planungsleistungen bei Grundschülern mit ADHS und LRS: Ein Vergleich von Fremdbeurteilungsverfahren und psychometrischen Testverfahren. Lernen und Lernstörungen, 4, 9-29.

Silver, C. H. (2014). Sources of data about children's executive functioning: Review and commentary. Child Neuropsychology, 20(1), 1 - 13. http://dx.doi.org/10.1080/09297049.2012.727793

Simmons, F. R., Willis, C. \& Adams, A.-M. (2012). Different components of working memory have different relationships with different mathematical skills. Journal of Experimental Child Psychology, 111 (2), 139 - 155. http://dx.doi.org/10.1016/j.jecp. 2011.08.011

Soto, E. F., Kofler, M.J., Singh, L. J., Wells, E. L., Irwin, L. N., Groves, N. B. et al. (2020). Executive functioning rating scales: Ecologically valid or construct invalid? Neuropsychology, 34(6), 605-619. http://dx.doi.org/10.1037/neu0000681 
Szúcs, D., Devine, A., Soltesz, F., Nobes, A. \& Gabriel, F. (2014). Cognitive components of a mathematical processing network in 9-year-old children. Developmental Sciene, 17(4), 506-524. http://dx.doi.org/10.1111/desc.12144

Tabachnick, B. G. \& Fidell, L. S. (2014). Using multivariate statistics (6 Aufl.). Harlow: Pearson.

Takacs, Z. K. \& Kassai, R. (2019). The efficacy of different interventions to foster children's executive function skills: A series of meta-analyses. Psychological Bulletin, 145(7), 653 - 697. http:// dx.doi.org/10.1037/bul0000195

Ten Eycke, K. D. \& Dewey, D. (2016). Parent-report and performance-based measures of executive function assess different constructs. Child Neuropsychology, 22(8), 889-906. http://dx. doi.org/10.1080/09297049.2015.1065961

Thorell, L. B. (2007). Do delay aversion and executive function deficits make distinct contributions to the functional impact of ADHD symptoms? A study of early academic skill deficits. Journal of Child Psychology and Psychiatry, 48(11), 1061-1070. http://dx.doi.org/10.1111/j.1469-7610.2007.01777.x

Titz, C. \& Karbach, J. (2014). Working memory and executive functions: Effects of training on academic achievement. Psychological Research, 78(6), 852-868. http://dx.doi.org/10.1007/s00 426-013-0537-1

Toplak, M. E., West, R. F. \& Stanovich, K. E. (2013). Practitioner review: Do performance-based measures and ratings of executive function assess the same construct? Journal of Child Psychology and Psychiatry and Allied Disciplines, 54(2), 131 - 143. http:// dx.doi.org/10.1111/jcpp.12001

Van der Ven, S. H., Kroesbergen, E. H., Boom, J. \& Leseman, P. P. (2012). The development of executive functions and early mathematics: A dynamic relationship. British Journal of Educational Psychology, 82, 100 -119. http://dx.doi.org/10.1111/j.20448279.2011.02035.x

Van der Ven, S. H., Kroesbergen, E. H., Boom, J. \& Leseman, P. P. (2013). The structure of executive functions in children: A closer examination of inhibition, shifting, and updating. British Journal of Educational Psychology, 31,70 - 87. http://dx.doi.org/10.1111/ j.2044-835X.2012.02079.x

Visu-Petra, L., Cheie, L., Benga, O. \& Miclea, M. (2011). Cognitive control goes to school: The impact of executive functions on academic performance. Procedia - Social and Behavioral Sciences, 11, 240 - 244. http://dx.doi.org/10.1016/j.sbspro.2011.01.069

Wegener, B. (1988). Kritik des Prestiges. Opladen: Westdeutscher Verlag.

Wirth, A., Reinelt, T., Gawrilow, C. \& Rauch, W. (2015). Selbstkontrolle in der Schule: Der Zusammenhang von geringer Selbstkontrolle und schlechten Schulleistungen bei Kindern mit ADHS. Lernen und Lernstörungen, 4, 245 -259. http://dx.doi.org/10. 1024/2235-0977/a000114
Yeniad, N., Malda, M., Mesman, J., van Ijzendoorn, M. H. \& Pieper, S. (2013). Shifting ability predicts math and reading performance in children: A meta-analytical study. Learning and Individual Differences, 23, 1-9. http://dx.doi.org/10.1016/j.lindif.2012. 10.004

Zorrilla-Silvestre, L., Presentación-Herrero, M. J. \& Gil-Gómez, J. (2016). The relationship between neuropsychological and ecological measurements of executive functioning in childhood and the prediction of mathematics performance. A pilot study. Electronic Journal of Research in Educational Psychology, 14(2), 333 - 351. http://dx.doi.org/10.14204/ejrep.39.15080

\section{Historie}

Manuskript eingegangen: 22.10.2020

Manuskript angenommen: 28.01.2021

Onlineveröffentlichung: 10.02.2021

\section{Autorenhinweis}

Teile des Datensatzes der Längsschnittstudie wurden bereits mit anderem inhaltlichen Fokus publiziert (Michel et al., 2020; Michel et al., 2019; Michel et al., 2018).

\section{Förderung}

Die Studie wurde von der Deutschen Forschungsgemeinschaft (DFG; Mi 1717/1-1) gefördert.

Open Access-Publikation ermöglicht durch die JuliusMaximilians-Universität Würzburg.

\section{ORCID}

Eva Michel

(D) https://orcid.org/0000-0001-8381-0348

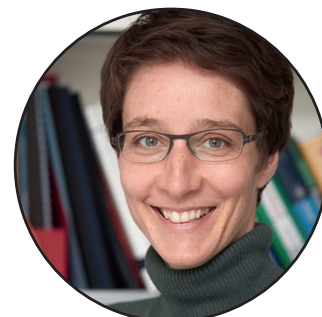

PD Dr. Eva Michel

Lehrstuhl für Psychologie IV Julius-Maximilians-Universität Würzburg Röntgenring 10 97070 Würzburg Deutschland eva.michel@uni-wuerzburg.de 